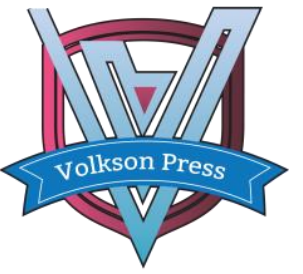

Contents List available at VOLKSON PRESS

Economics \& Management Innovations(EMI)

DOI : http://doi.org/10.26480/icemi.01.2017.46.48

\title{
Methods of Improving the Recall and Precision of Sci-tech Novelty Search
}

\author{
Wang $\mathrm{Li}^{1}$, Liu Wen ${ }^{1}$

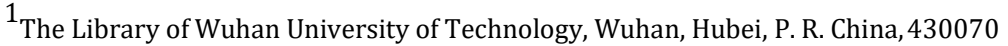 \\ *Email: naonao586@163.com
}

This is an open access article distributed under the Creative Commons Attribution License, which permits unrestricted use, distribution, and reproduction in any medium, provided the original work is properly cited.

\section{ARTICLE DETAILS}

\section{Article History:}

Received 02 october 2017 Accepted 06 october 2017 Available online 11 october 2017

\section{Keywords:}

Sci-tech Novelty Search, Recall Rate, Precision Rate.

\section{ABSTRACT}

Sci-tech novelty search is a kind of information consulting service aiming at testifying the novelty and advancement of the research project, and it will make use of various research and analysis methods including information search and comparative analysis of literature. In the process of conducting novelty search, the recall rate and the precision rate will be used to indicate the relevance and accuracy of novelty retrieving points respectively, which are considered to be important indexes of evaluating the quality of novelty search report. In this essay, it focuses on the issue of recall and precision of information Search in the process of conducting sci-tech novelty search. By combining with the practical case analysis, it puts forwards effective methods of improving the recall and precision of sci-tech noveltysearch.

\section{Introduction}

Sci-tech novelty search is a kind of information consulting service aiming at testifying the novelty and advancement of the research project, and it will make use of various research and analysis methods including information search and comparative analysis of literature ${ }^{[6]}$. The correct evaluation and judgment on the novelty of research project will pose great influence on the quality of novelty search report. In the process of conducting novelty search, the recall rate and the precision rate will be used to indicate the relevance and accuracy of novelty retrieving points respectively, which are considered to be important indexes of evaluating the quality of novelty search report.The recall rate is used to evaluate the capacity and effectiveness of information searching system in retrieving relevant literature ${ }^{[3]}$. The precision rate is used to evaluate the accuracy of the information searching system in retrieving relevantliterature.

Recall Rate $=\frac{\text { Number of retrived literature that are relevant to the novelty retrieval point }}{\text { Total number of relevant literature in the system }}$

$$
\text { Total number of relevant literature in the system }
$$

Precision Rate $=\frac{\text { Number of retrived literature that are mostly relevant to the novelty retrieval point }}{\text { Nutrieval point }}$

In order to guarantee the quality of novelty search report on certain research topics, it is necessary for novelty researchers to pay more attention to improving the recall rate and precision rate of literature search. In this essay, it will discuss methods of improving the recall rate and precision rate of sci-tech novelty search from the following aspects.

\section{Proper Selection of Database}

It is of great importance for novelty researchers to gain an overall understanding of the content and embody range of database and flexibly choose from them. By making use of database with high quality citation, large number of embodying articles, wide time coverage and fast updating frequency, the recall rate could be guaranteed to a large extent.

\subsection{Large and Comprehensive Database}

In the process of conducting domestic novelty search, some comprehensive databases could be utilized. China Journal Full-text Database is a part of China National Knowledge Infrastructure (CNKI), which is managed by Tsinghua Tongfang Co,. Ltd. It is the largest China journal full text database with continuous updating. There are more than 8 million fulltext articles and 15 million bibliographies that are classified into nine major specials and 126 special document database. The database collects literature ever since 1994 with more than 6,100 core journals and a wide embody range including Science and Technology A (mathematics, science, chemistry, astronomy, geosciences and biology), Science and Technology $\mathrm{B}$ (chemistry and chemical engineering, energy and materials), Science and Technology C (industrial technology), Agriculture, Medicine Science, Literature, History and Philosophy, Economic, Politics and Law, Education and Social Science, Electronic Technology and Information Science. The China Journal Full-text Database is characteristic of its wide coverage range and fast updating speed. By conducting daily updating in the core website of China National Knowledge Infrastructure and database exchange service center, it is possible for the mirror sites to update transmission data every day through the Internet or satellite, update science and technology subject CDs every month, update literature, history and philosophy subject CDs every two months, and update thematic CDs once a year. Secondly is the China Science and Technology Full-text Journal Database published by Chongqing VIP Information Co., Ltd. It collects all the Chinese journals including 1,957 types of core journals including subjects such as social science, natural science, engineering and technology, agricultural science, medicine science, economic management, education science, library and information science. Lastly is the Digitalized Journal Full-text Database managed by Wan Fang Data system, which collects 4,616 kinds of journals by combining China Science and Technology Paper, Citation Database and other relevant databases. It generally covers all kinds of natural science journals and social science core journals in China.

For domestic and international novelty search, foreign comprehensive databases could be applied. For instance, Engineer Index is a large comprehensive database from the United States, covering a variety of engineering technology fields and its management such as mechanics, electronics, light industry, petroleum, architecture and municipal engineering. In the process of researching some applied science or engineering projects with intensified technology, EI will be of great help for novelty researchers. Web of Science is a product developed by Thomson Scientific Information Corporation based on the WEB development, which is known as a comprehensive and multidisciplinary database for the core journal citation index database[4]. It consists of three main citation databases including Science Citation Index (SCI), Social Science Citation Index (SSCI) and Arts \& Humanities Citation Index (A\&HCI), two chemistry information factual databases including Current Chemical Reactions (CCR) and Index Chemicus (IC) as well as three citation databases including Science Citation Index Expanded (SCIE), Conference Proceedings Citation Index-Science (CPCI-S) and Conference Proceedings Citation index-Social Science \& Humanalities (CPCI-SSH). It collects more than 10,000 high quality and influential academic journals in the world with a coverage of natural science, engineering technology, social science, art and humanities. Among these databases, the online edition SCIE collects more than 8,800 journal abstracts 
and citations covering fields such as natural science, engineering technology, mathematics, physical, materials and chemistry with data updating every week. As for SSCI, it collects more than 3,200 kinds of world top class social science journals. The All Covered Journals of Art \& Humanities Citation Index collects 1,700 kinds of world top class social science journals

\subsection{Professional Database}

Chemical Abstracts (CA) acts as the search tool in the field of chemistry and engineering. It collects professional literature including the chemical engineering and biological literature such as chemical components of materials, formulations, preparation technology and manufacturing methods. In order to tackle with literature demands from different subjects and fields, relevant professional databases have been developed. For instance, in the Wuhan University of Technology, a "Literature Collection for Key Subjects in Wuhan University of Technology" has been established to inspire and support the independent innovation in characteristic subjects in Wuhan University of Technology. It includes school key subjects such as naval architecture and marine engineering, control science and engineering, information and communication engineering, mechanical engineering, materials science and engineering, chemistry, chemical engineering and life science. By constructing the relevant literature database according to the subject features and development requirements of Wuhan University of Technology, it provides assistance for researchers in Wuhan University to conduct scientific research and literature search.

\section{Formulation of Proper Search Strategy}

The search strategy is generally guided by the improvement of recall rate, on the basis of which the precision rate should also be improved in avoidance of missed search
Boolean combination and search formula would pose negative Boolean combination and search formula would pose negative
influences on the comprehensiveness and precision of search result. Generally speaking, search strategies include the extract of core search key words and the formulation of search formula ${ }^{[2]}$.

\subsection{The Selection of Precise Search Key Words}

When the search words fail to indicate certain fields of the research topic, it could not precisely reflect the novelty points and search requirements. Therefore, the selection of proper search key words plays an important role to improve the recall rate and precision rate of the novelty search. Some of the widespread methods of selecting search words are as following.

\subsubsection{Synonym Search}

For instance, synonyms of "computer" include "microcomputer" and "pc", and synonyms of "colleges" include "universities" and "institutions of higher learning". In the domestic and international novelty search, there are usually more than one English search words in equivalent with Chinese word, which lays emphasis on the overall consideration of synonym words in avoidance of missed search.

\subsubsection{Truncation search}

In order to expand the search scope and avoid missed search, truncation search could be applied by adding wildcard "?" or truncation symbol "*” in the front, middle or end of search words to reach the goal of searching a group of words with similar concepts or the same root. Wildcard "?" could only replace one character. For example, the search word of "re?d" could lead to search results such as "read" and "reid". As for the truncation symbol "*", it can be used to indicate limitless truncation words by searching fields with different meanings. By applying wildcards or truncation symbols, it is possible to expand the search scope and increase the recall rate of search. For instance, in the case of conducting domestic novelty search on "NL tester", none relevant literature has been retrieved based on the key word of "NL tester" in CNKI. However, when the search word is replaced by "* tester", a large number of relevant literature could be retrieved. In the process of retrieving international literature, truncation symbol "*" could be added to the end of search word to make sure all parts of speech and derivative words could be retrieved. For instance, the search word of "chem*" could retrieve relevant literature such as "chemic", "chemical" and "chemistry".

\subsubsection{Abbreviation and Element Symbol Search}

For instance, "CAD" could be used to retrieve "computer aided design", "SCC" could be used to retrieve "self-compacting concrete" and "CO2" could be used to retrieve "carbon dioxide". Abbreviation and element symbol search have been widely applied in retrieving chemical substances' names and abbreviations, which calls for the familiarity with abbreviation of professional names and element symbols of relevant chemical substances.

\subsubsection{Extended Concept Search}

Professional nouns and terms are widespread in search, which means that insufficient search strategies of key words will lead to missed information. For example, if conduct precise search with " $(\mathrm{Ca}(\mathrm{OH}) 2)$ " as the key word in China Journal Website, the search results are 4,916 articles. However, "slaked lime" is found out to be the byname of " $(\mathrm{Ca}(\mathrm{OH}) 2)$ ". Therefore, by conducting precise search with " $(\mathrm{Ca}(\mathrm{OH}) 2)$ OR (slaked lime)" as the key word, 5,525 relevant articles are found, which is far more than the former search results. As a result, it might be concluded that extended information such as bynames of key words should be paid with more attention to avoid missed information.

\subsubsection{Superior and subordinate key word search.}

Superior key word refers to a wider range of concepts and connotations. Subordinate key word refers to specified concepts with narrower connotations. In order to improve the recall rate, both superior and subordinate key words should be applied in search including synonyms, bynames and professional terms.

Search researchers tend to make use of subordinate key words in working out search strategies to guarantee the precision rate. However, in order to avoid missed information, superior key words should be added to make sure the recall rate of search $[8]$. Take the
novelty search task "Research on the transmission dynamics structure and function of silver halide series fast ionic conductor " as an example. Silver halides include $\mathrm{AgCl}, \mathrm{AgBr}$ and $\mathrm{AgI}$, which are representative examples of fast ionic conductors. Therefore, the subordinate key words include $\mathrm{AgCl}, \mathrm{AgBr}$ and $\mathrm{AgI}$, and the regular search strategy could be ((silver halides)AND(power) $\operatorname{AND}($ (construction) OR(performance)), and the search result is one relevant article. However, when combine superior and subordinate key words in extended search with ((AgCl) OR (AgBr) OR (AgI) OR(fast ion conductor) $\mathrm{OR}($ silver halides)) $\mathrm{AND}($ (construction) OR(performance)), there are 27 relevant articles with 26 newly retrieved articles. It might be concluded that the combination of both superior and subordinate key word search would contribute to the recall rate the novelty search.

\subsection{Formulation of search expressions based on the goal of novelty search}

In the process of conducting novelty search on result evaluation, the search strategy should cover the narrow and broad terms to improve the precision rate. When there is no relevant literature or a small number of it, the search expressions and conditions should be adjusted to expand the search coverage range and gain access to more literature to make sure the precision rate. Otherwise, the novelty search with high recall rate but low precision rate will negatively affect the evaluation on novelty of the search research project and its quality. On the other hand, when the subject of novelty research is too broad with many retrieved articles, more careful and thorough analysis on the technology points of novelty research should be conducted to extract precise key words. By narrowing the search range including the application fields, composition of materials and research methods, it is possible to formulate a more precise novelty search strategy and search more relevant literature.

As for the novelty search on research projects, both novelty researchers and users should gain a good knowledge of the research progress to meet the high requirement on recall rate. Only by basing on all the relevant literature in the database could it possible to overall understand the research progress of the novelty research projects and make proper judgment. The formulation of search expression should follow the order of broad to narrow by adding more search key words until the novelty researcher gain a satisfied search result.

\subsection{Proper Application of Logical Operators}

Logical operators are also known as Boolean operators, which include "OR","AND" and "NOT".

(1) Logical operator "OR" (+) is usually used to make collocation between synonyms and related words. The search expression could be "A OR B" or "A+B", which is characteristic of higher recall rate compared with former collocation by extending the search range and improving the recall rate. 
connections between different concepts and pose search restrictions to narrow down the search range, reduce retrieved literature and improve the precision rate of novelty search. For instance, the search expression of "(light industry) AND (textile industry)" will lead to literature involved with both "light industry" and "textile industry".

(3) Logical operator "NOT" (-) is usually used to remove unnecessary of unrelated key words. The Application of "NOT" is considered to contribute to the narrowing of search range, reduce amount of information and improve the precision rate.

\section{Development of High Quality Novelty Search Researchers}

The level of professional knowledge and the working attitude of novelty search researchers will pose a direct influence on the quality of novelty search report. The quality of sci-tech novelty search researchers plays a key role in improving the quality and effectiveness of sci-tech novelty search. A qualified sci-tech novelty search researcher should be equipped with good knowledge in the professional field, computer and English, as well as good psychological personalities such as dedication to work, pragmatism and spirit of exploration. It requires the constant devotion to learning and self improvement of novelty search researchers, thus reaching the goal of improving the recall rate and precision rate and establishing an overall knowledge structure that could adapt to the requirements of sci-tech novelty search task [1]. For novelty search researchers with less experience, they should work together to optimize their advantage knowledge background and complete the novelty search task.

In conclusion, in order to improve the recall rate and precision rate of sci-tech novelty search, novelty search researchers should be equipped with corresponding professional knowledge and qualifications to grasp the key points of novelty and understand the whole novelty search topic. By properly selecting database and search key words, formulating effective search strategies and utilizing various search methods, it is possible for novelty search researchers to reach the novelty search goal and guarantee its quality.

\section{Bibliography}

Wang Li (1982-), female, postgraduate of 2008 from Wuhan University of Technology, School of Automation, now works in the library of Wuhan University of Technology, novelty search station. Corresponding author: Liu Wen (1983-), female, postgraduate of 2008 from Wuhan University of Technology, School of Materials Science and Engineering, now works in the library of Wuhan University of Technology, novelty search station.

\section{References}

[1] Long Zeyun, et al. in: Methods of Improving the Recall Rate and Precision Rate of Literature Database, edtied by Journal of Intelligence, 1996, (2).

[2] Ma Jingdi, et al. in: Effectiveness of Search Strategy in Novelty Search. edtied by Library and Information Service, 1997, (2).

[3] Zhang Qifang. in: Methods of Improving Recall Rate in Novelty Search. edtied by Journal of Intelligence, 1999, (7).

[4] Ma Jianchun. in: Analysis on Methods of Improving Recall Rate in Sci-tech Novelty Search. edtied by 2010, (20).

[5] Wu Qiye. in: Differences in Recall Rate and Precision Rate in Scitech Novelty Search and Literature Search [J]. 2003, (9).

[6] Dong Yueling. in: Research on the Value of Sci-tech Novelty Search in Universities. Library \& Information Service of Zhejiang Universities \& Colleges [J]. 2011, 29 (2):3-6.

[7] Hongyan. in: Impacts Psed by the Formulation of Search Strategy and Expression on the Quality of Sci-tech Novelty Search . edtied by Journal of Science of Teachers' College and University, 2009, 29(3):75-77.

[8] Hao Hui, Hu Juan. in: Impacts Posed by Process of Sci-tech Novelty Search on Quality of Novelty Search. edtied by Journal of Modern Information, 2015, 35(5):149-152. 